\title{
A clinical study of lung cancer dose calculation accuracy with Monte Carlo simulation
}

\author{
Yanqun Zhao, Guohai Qi, Gang Yin, Xianliang Wang, Pei Wang ${ }^{*}$, Jian Li, Mingyong Xiao, Jie Li, Shengwei Kang \\ and Xiongfei Liao
}

\begin{abstract}
Background: The accuracy of dose calculation is crucial to the quality of treatment planning and, consequently, to the dose delivered to patients undergoing radiation therapy. Current general calculation algorithms such as Pencil Beam Convolution (PBC) and Collapsed Cone Convolution (CCC) have shortcomings in regard to severe inhomogeneities, particularly in those regions where charged particle equilibrium does not hold. The aim of this study was to evaluate the accuracy of the PBC and CCC algorithms in lung cancer radiotherapy using Monte Carlo (MC) technology.

Methods and materials: Four treatment plans were designed using Oncentra Masterplan TPS for each patient. Two intensity-modulated radiation therapy (IMRT) plans were developed using the PBC and CCC algorithms, and two three-dimensional conformal therapy (3DCRT) plans were developed using the PBC and CCC algorithms. The DICOM-RT files of the treatment plans were exported to the Monte Carlo system to recalculate. The dose distributions of GTV, PTV and ipsilateral lung calculated by the TPS and MC were compared.

Result: For 3DCRT and IMRT plans, the mean dose differences for GTV between the CCC and MC increased with decreasing of the GTV volume. For IMRT, the mean dose differences were found to be higher than that of 3DCRT. The CCC algorithm overestimated the GTV mean dose by approximately 3\% for IMRT. For 3DCRT plans, when the volume of the GTV was greater than $100 \mathrm{~cm}^{3}$, the mean doses calculated by CCC and MC almost have no difference. PBC shows large deviations from the MC algorithm. For the dose to the ipsilateral lung, the CCC algorithm overestimated the dose to the entire lung, and the PBC algorithm overestimated $V_{20}$ but underestimated $V_{5}$; the difference in $V_{10}$ was not statistically significant.

Conclusions: PBC substantially overestimates the dose to the tumour, but the CCC is similar to the MC simulation. It is recommended that the treatment plans for lung cancer be developed using an advanced dose calculation algorithm other than PBC. MC can accurately calculate the dose distribution in lung cancer and can provide a notably effective tool for benchmarking the performance of other dose calculation algorithms within patients.
\end{abstract}

Keywords: 3-Dimensional conformal radiation therapy, Collapsed cone convolution, Pencil beam convolution, Lung cancer, Monte Carlo, Intensity-modulated radiation therapy

\section{Introduction}

The accuracy of dose calculation is crucial to the quality of treatment planning and, consequently, to the dose delivered to patients undergoing radiation therapy [1]. In the past 20 years, radiotherapy has become increasingly complex. Complex treatments such as intensitymodulated radiation therapy (IMRT) are expected to provide better treatment outcomes for patients and better

\footnotetext{
* Correspondence: dengwangpei@163.com

Department of Radiation Oncology, Sichuan Provincial Cancer Hospital,

Chengdu, Sichuan 610041, China
}

sparing of healthy tissues [2]. The increased complexity of the delivery and dosimetry of radiotherapy treatments arising from the increasing use of IMRT treatments has led to an increased demand for accurate treatment verification [2]. The International Commission on Radiation Units and Measurements (ICRU) recommends that patient-specific independent treatment plan verification should be performed for all IMRT treatments [3].

There is general agreement that, in IMRT, the actual delivered dose (or location) should be within 3\% (or $3 \mathrm{~mm}$ ) of the planned TPS [4]. But what is the actual dose? We

\section{Biomed Central}

2014 Zhao et al.; licensee BioMed Central. This is an Open Access article distributed under the terms of the Creative Commons Attribution License (http://creativecommons.org/licenses/by/4.0), which permits unrestricted use, distribution, and reproduction in any medium, provided the original work is properly credited. The Creative Commons Public Domain Dedication waiver (http://creativecommons.org/publicdomain/zero/1.0/) applies to the data made available in this article, unless otherwise stated. 
need to know if the plan delivered is an accurate representation of the calculated plan. Patient-specific pre-treatment quality assurance can be performed using film, ion chamber and diode arrays. But most of Patient-specific QA were performed on phantom, which will lead to a lack of predictive power for clinically relevant patient dose errors. [5] The ICRU Report 83 recommends that as an alternative to a set of measured absorbed-dose distributions, it is acceptable to use independent absorbed-dose calculations instead of performing measurements. Currently, independent absorbed-dose calculations have been performed in many radiotherapy centres, but some of them use simple correction-based dose verification calculations, which are known to be less accurate than the treatment planning algorithms that they are designed to verify [6]. The use of inferior standards for QA procedures might lead to high false-negative or false-positive rates. Therefore, the accuracy of the independent absorbed-dose calculation which was used to evaluate the IMRT algorithm should be equivalent or higher than that of the treatment-planning system. A Monte Carlo algorithm would be acceptable for determining the absorbed dose in the presence of inhomogeneous tissue if the Monte Carlo code is tested sufficiently [3].

In routine clinical applications, calculations of dose to the tumour are performed by commercial treatment planning systems (TPS). The majority of these systems employ a Pencil Beam Convolution (PBC) algorithm for dose calculation. This algorithm is commonly used in clinical practice because it is very fast, but it is widely known that $\mathrm{PBC}$ has shortcomings in regard to severe inhomogeneities, particularly in those regions where charged particle equilibrium does not hold. This is especially questionable for target dose calculations in lung cancer treatments. In some previous studies, the prediction of $\mathrm{PBC}$ deviates from the measured values by as much as $15 \%[7,8]$. Another algorithm, the Collapsed Cone Convolution (CCC), is utilised in commercial treatment planning systems, and the CCC algorithm produces values that are closer to the measured values than the PBC algorithm $[9,10]$ but still deviates from the measurement by more than $5 \%$ under certain circumstances [7,8,11-13].

Monte Carlo (MC) simulation has proved to be the most accurate dose calculation and is therefore used to evaluate other dose calculation algorithms [7,8,11-16]. The MC method is potentially highly accurate as it can faithfully model both photon scatter and electron transport in arbitrary materials. Although benchmarking of commercial TPS dose calculations should ideally be performed against measured dose distributions, situations can occur where measurement cannot be made with high accuracy; in such situations, the MC algorithm is used as a reference against which TPS calculations can be benchmarked [17].
Although comparisons of MC calculation with the model-based calculations were reported elsewhere $[12,13]$, great majority of previous studies have been performed for a phantom study or a specific site, and to our knowledge few studies has been devoted to intensity modulated radiation (IMRT) for clinical lung cancer. In this study, we will compare the dose distributions for 24 lung cancer patients, including the dose distributions calculated by MC, CCC and PBC for 3DCRT and IMRT.

\section{Materials and methods}

\section{Patients and treatment planning}

A total of 24 lung cancer patients, who had been previously treated at our hospital, were randomly selected for this study. The tumour size and location varied from patient to patient. The treatment plans were based on the patient CT scanned in a supine position under normal free breathing conditions. Mean grass tumour volume (GTV) size was $68.9 ? 56 \mathrm{~cm}^{3}$ (range, $5 \sim 162 \mathrm{~cm}^{3}$ ) and mean planning target volume (PTV) size was 133.9 ? $99.2 \mathrm{~cm}^{3}$ (range, $21 \sim 305.7 \mathrm{~cm}^{3}$ ). The Oncentra Masterplan V4.1 treatment planning system was used for the plan dose calculation. The Oncentra Masterplan TPS employs two algorithms, the Pencil Beam Convolution (PBC) and the Collapsed Cone Convolution (CCC).

Four plans were designed for each patient, two 3DCRT plans, one that used the CCC and one that used the $\mathrm{PBC}$, and two IMRT plans, one of which that used the $\mathrm{CCC}$ and one that used the $\mathrm{PBC}$ for plan optimizations and final dose calculations, respectively. To facilitate the comparison, the parameters of the two IMRT plans were the same, and the conditions of the two 3DCRT plans were identical. The calculation grid size was $0.3 \mathrm{~cm}$ ? $0.3 \mathrm{~cm}$ ? $0.3 \mathrm{~cm}$ for all plans. For IMRT plans, the minimum open field size was $4 \mathrm{~cm}^{2}$ and minimum MU per segment was $3 \mathrm{MU}$, and the delivery type was step-andshoot. For all of the plans, the prescribed dose was $60 \mathrm{~Gy} /$ $30 \mathrm{Fx}$, and all plans were normalized so that $95 \%$ of PTV received $\geq 100 \%$ prescribed dose. Radiation treatments were delivered on an $8 \mathrm{MV}$ Varian $23 \mathrm{EX}$ linear accelerator equipped with a multileaf collimator (60 pairs, the minimum leaf width projection into the iso-centre Plane is $5 \mathrm{~mm}$ ). The patients CT and the patients RTPLAN files were exported to the $\mathrm{MC}$ work station to recalculate the dose distribution.

\section{Monte Carlo calculation}

This study was performed using BEAMnrc [18], DOSXYZnrc [19] and a well-commissioned in-house Monte Carlo code MCSIM [20] which came from the Fox Chase Cancer Center. MCSIM is a system based on EGS4, which accepts standard DICOM-RT files exported from a commercial treatment planning system and produces $\mathrm{MC}$ calculated dose distributions. 
The accuracy of the Monte Carlo model for the Varian 23EX linear accelerator $8 \mathrm{MV}$ photon beam employed in this investigation has been thoroughly tested. In this model, the phase-space source that has been fine-tuned was used to reproduce dose profiles and percent depth doses (PDD) in water phantom. The dose profiles curves and percent depth doses curves from Monte Carlo calculation were compared to the measurements, and they were in good agreement with measurements.

The accuracy of phase-space source also tested and verified in a solid water phantom. A solid water phantom with a virtual tumor was employed in our study, and a sevenfield (10? $10 \mathrm{~cm}^{2}$ open fields) 3DCRT treatment plan was created by TPS for a tumor which diameter was $8 \mathrm{~cm}$. The plan was calculated using CCC and was imported in a DICOM-RT format from TPS to the MC system to recalculate. Dose distributions and cumulative dose-volume histograms (DVHs) in the water-equivalent phantom calculated by $\mathrm{CCC}$ and $\mathrm{MC}$ are shown in Figure 1. From the figure we know that the dose distributions calculated by CCC and MC agreed very well. In the same way, four 3DCRT treatment plans which field sizes were 5 ? 5, 15 ? 15,20 ? 20, 25 ? $25 \mathrm{~cm}^{2}$, respectively, for corresponding virtual tumor which diameter were $3,12,18$ and $22 \mathrm{~cm}$ were all recalculated using $\mathrm{MC}$ and compared with corresponding TPS calculations, the discrepancies in the corresponding target dose were within $1 \%$.

In this study, the Monte Carlo calculation grid size was $0.2 \mathrm{~cm} ? 0.2 \mathrm{~cm} ? 0.3 \mathrm{~cm}$, the ECUT $=0.7 \mathrm{MeV}$, PCUT = $0.01 \mathrm{MeV}$. In all patient calculations, we have kept the statistical uncertainty to be $2 \%$ or less so as not to significantly affect isodose lines, DVHs, or biological indices [21].
The absolute dose was calculated by converting the $\mathrm{MC}$ calculated dose per fluence to the dose per MU under linac calibration conditions in water (depth of $2 \mathrm{~cm}, 10$ ? $10 \mathrm{~cm}^{2}$ field size, $100 \mathrm{~cm} \mathrm{SSD}$, and $100 \mathrm{cGy}$ for $100 \mathrm{MU})$.

\section{Lung phantom dose measurement}

The QUASAR multi-purpose body phantom was employed to model a patient thorax: it is a $30 \mathrm{~cm}$ wide, $12 \mathrm{~cm}$ long and $20 \mathrm{~cm}$ high acrylic body oval; with openings for cylindrical inserts of $8 \mathrm{~cm}$ and $2 \mathrm{~cm}$ diameter. These openings allow the placement of ion chambers for measurement. Two $8 \mathrm{~cm}$ diameter cedar wood cylindrical inserts were placed into the openings, approximately representing lung tissue, and one $2 \mathrm{~cm}$ diameter tumour-equivalent rod was inserted with the points of measurement. The phantom is shown in Figure 2. In the QUASAR multipurpose body phantom, two 3DCRT plans with 5 fields were designed for the $2 \mathrm{~cm}$ diameter tumour using the Oncentra Masterplan treatment planning system. One plan was calculated using PBC and the other was calculated using CCC, the plan prescribed dose for 95\% volume of tumour were $200 \mathrm{cGy}$. The treatment plans DICOM-RT files were imported into the MC system to recalculate, and the two treatment plans were also delivered on linac. An IBA CC13 ion chamber was used for dose measurement.

\section{Statistical analysis}

The percent difference between the TPS algorithms and the MC simulations were compared for all plan evaluation parameters. Pairwise comparisons were performed
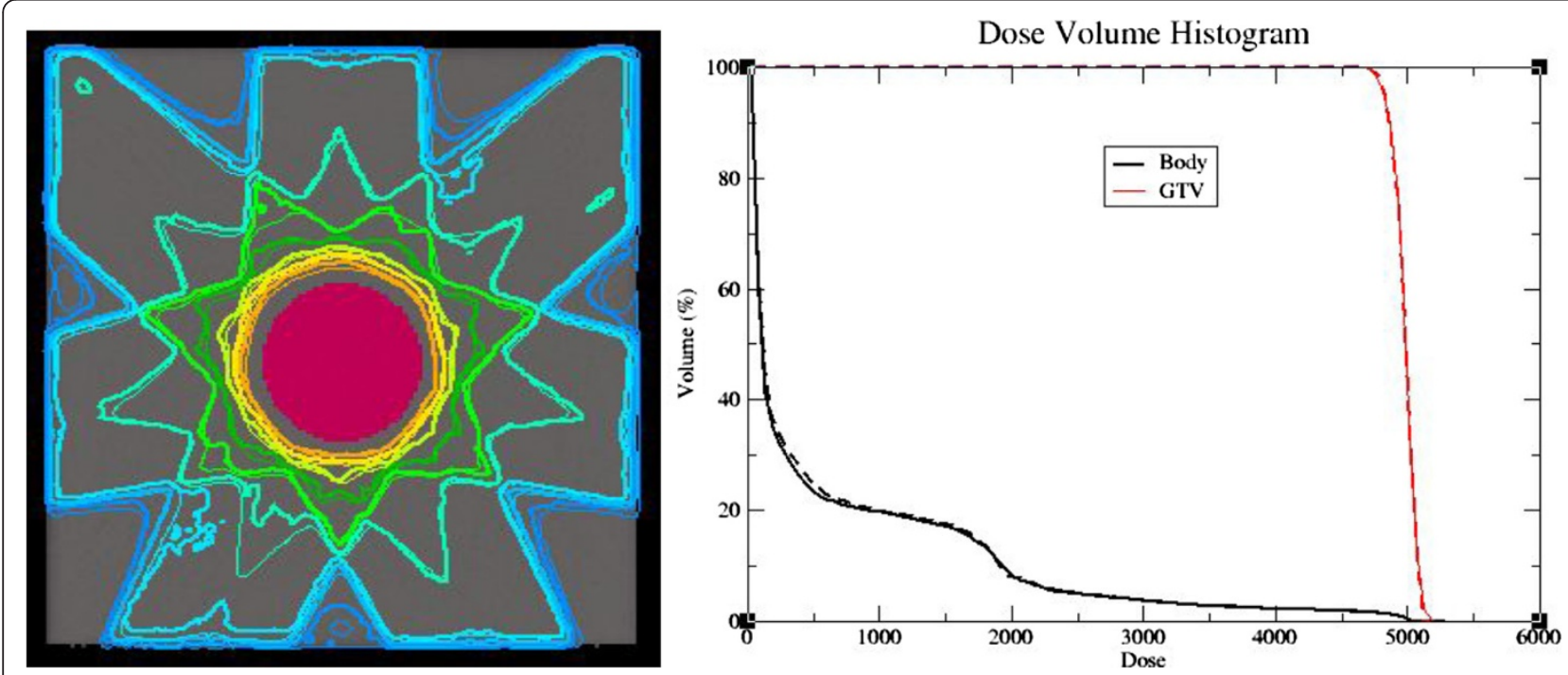

Figure 1 Comparison of dose distributions and DVHs calculated using CCC and MC for a $8 \mathrm{~cm}$ diameter virtual tumor in solid water phantom. On the left are the dose distributions, thick line represents MC and thin line represents TPS, On the right are the DVHs, solid line represents TPS and dotted line represents MC. 


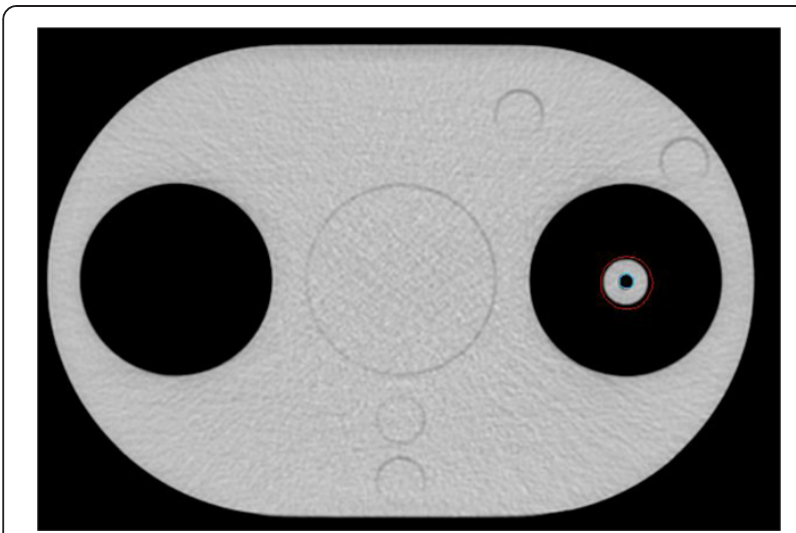

Figure 2 QUASAR lung phantom with ion chamber in the tumor insert.

using the paired t-test. $\mathrm{P}<0.05$ was considered significant. A linear regression model was used to test correlation between the percent differences in plan evaluation parameters. $R^{2}$ values were used to evaluate goodness of fit of the linear models.

\section{Results}

\section{Lung phantom dose measurement}

In the QUASAR multi-purpose body phantom, the dose to the tumour centre was compared among all the calculations and measurements. The results of the TPS calculations, the MC calculations and the ion chamber measurements are listed in Table 1 . The table reveals that the MC calculation doses are very close to the measurements, and the discrepancies between the MC calculations and the measurements are within $1 \%$.

\section{Clinical study}

Dose distributions and plan evaluation parameters calculated with the $\mathrm{MC}$ algorithm were compared to the $\mathrm{PBC}$ and $\mathrm{CCC}$ algorithms. The $\mathrm{PBC}$ and $\mathrm{CCC}$ result minus the $\mathrm{MC}$ result was expressed as a percentage. Dose-volume histograms (DVHs) were created for each patient. The GTV, PTV and ipsilateral lung were compared. Due to their clinical utility for predicting OAR toxicity, the dosevolume points were used for comparison. For lung, the dose-volume points $\mathrm{V}_{5}, \mathrm{~V}_{10}$, and $\mathrm{V}_{20}$ (the lung volume that receives at least 5,10 and $20 \mathrm{~Gy}$, respectively) were used. To evaluate the differences in the stability of the dose- volume points that denote target coverage, the dose volume points near-minimal dose $\left(\mathrm{D}_{98}\right), \mathrm{D}_{95}, \mathrm{D}_{90}, \mathrm{D}_{50}$, nearmaximum dose $\left(D_{2}\right)$ (dose received by $98,95,90,50$, and $2 \%$ of the volume, respectively) were compared.

Figure 3 shows the comparisons of dose distributions and DVHs of a lung cancer patients four treatment plans. The dose distributions are on the left and the DVHs are on the right. In general, CCC calculations are closer to $\mathrm{MC}$ calculations for this patient; the PBC calculations exhibit large deviations from the $\mathrm{MC}$ calculations.

Table 2 and Table 3 show the results of the statistical analysis of GTV, PTV and ipsilateral lung for all 24 patients, and the corresponding $\mathrm{P}$ values are listed in the table.

Figure 4a shows the mean dose differences of GTV between CCC and MC. For 3DCRT, the mean dose differences for the GTV decreases with increasing of the GTV volume; when the volume of the GTV was greater than $100 \mathrm{~cm}^{3}$, the mean doses calculated by CCC and $\mathrm{MC}$ almost have no difference. For the IMRT plans, the mean dose differences were larger than that of the 3DCRT plans. As shown in Table 2, the mean dose difference (CCC vs. MC) in the GTV for IMRT is $3.43 \%$ and $3.05 \%$ for the PTV. The mean dose difference (CCC vs. MC) in the GTV for 3DCRT is $0.62 \%$ and $0.42 \%$ for the PTV. P $>0.05$ indicates that the difference between the CCC and MC for the 3DCRT plans is not statistically significant.

PBC shows large deviations from MC. Figure $4 \mathrm{~b}$ shows that the mean dose differences for GTV between the $\mathrm{PBC}$ and $\mathrm{MC}$ increases with decreasing GTV volume for both 3DCRT and IMRT. PBC overestimated the mean dose of GTV and PTV, and when the volume of GTV was less than $10 \mathrm{~cm}^{3}$, the difference increases substantially, the maximum difference in the mean dose for individual patients was as high as $19 \%$. As shown in Table 2, the mean dose difference (PBC vs. MC) in the GTV for 3DCRT plans was $5.95 \%$ and $7.96 \%$ for the PTV, and the mean dose difference (PBC vs. MC) in the GTV for IMRT plans is $7.81 \%$ and $10.23 \%$ for the PTV.

From Table 2 we know that the difference of $\mathrm{D}_{98}$ between CCC and MC is the largest among all dose-volume points in IMRT, and the difference become smaller gradually from $D_{98}, D_{95}, D_{90}, D_{50}$ to $D_{2}$, the effect is not obvious in 3DCRT. The differences of dose-volume points

Table 1 Results of dose comparison for the tumour centre among the TPS calculations and the MC simulations and measurements

\begin{tabular}{|c|c|c|c|c|c|c|c|c|c|c|c|}
\hline \multicolumn{6}{|c|}{ CCC algorithm evaluation } & \multicolumn{6}{|c|}{ PBC algorithm evaluation } \\
\hline Meas & $\mathrm{CCC}$ & MUs & Diff $_{\text {CcC-Meas }}$ & MC & Diff $_{\text {MC-Meas }}$ & Meas & PBC & MUs & Diff $_{\text {PBC-Meas }}$ & MC & Diff $_{\text {MC-Meas }}$ \\
\hline 203.1 & 199.6 & 250 & $-1.7 \%$ & 202.6 & $0.2 \%$ & 194.6 & 200.5 & 240 & $3.0 \%$ & 195.8 & $0.6 \%$ \\
\hline
\end{tabular}

Note: CCC = Collapsed Cone Convolution; PBC = Pencil Beam Convolution; MC = Monte Carlo; Meas = measurement. 

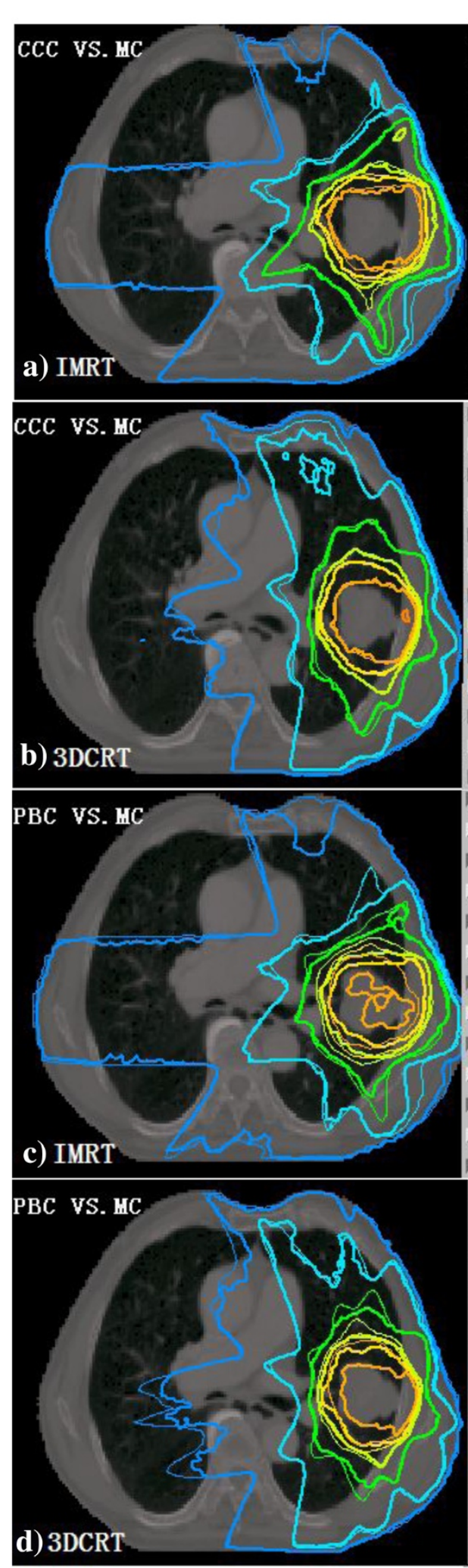
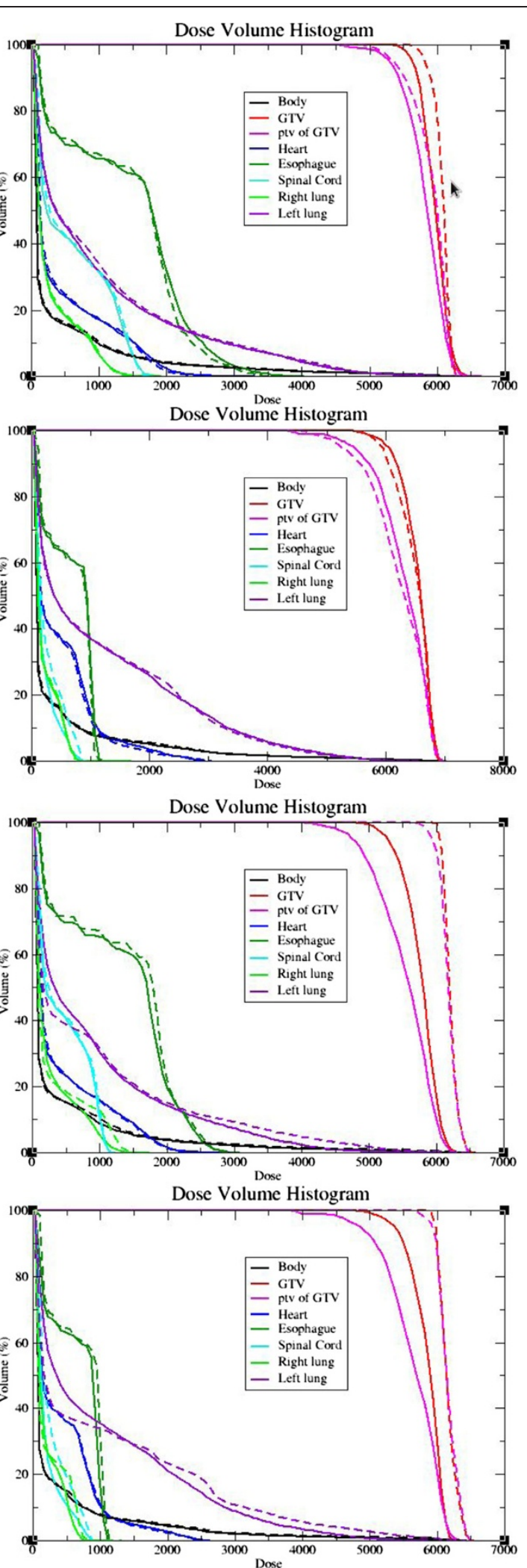

Figure 3 (See legend on next page.) 
(See figure on previous page.)

Figure 3 Comparison of the dose distributions and DVHs of a lung cancer patient s four treatment plans; on the left are the dose distributions, the thin line represents the TPS, the thick line represents MC, on the right are the DVHs corresponding to the left, dotted line represents TPS and solid line represents MC. a) IMRT plans calculated using CCC and MC recalculation, b) 3DCRT plans calculated using CCC and MC recalculation c) IMRT plans calculated using PBC and MC recalculation d) 3DCRT plans calculated using PBC and $M C$ recalculation,

for GTV between PBC and MC for all individual patients become smaller gradually from $D_{98}, D_{95} 、 D_{90} 、 D_{50}$ to $\mathrm{D}_{2}$ for IMRT plans too, the effect is less pronounced for 3DCRT. In general, the differences of dose-volume points for GTV between $\mathrm{PBC}$ and $\mathrm{MC}$ are larger than that of $\mathrm{CCC}$ and MC.

Table 3 shows the results of the statistical analysis for the ipsilateral lung. As shown in the table, the CCC algorithm overestimated the dose for the entire lung, overestimated $\mathrm{V}_{5}, \mathrm{~V}_{10}$, and $\mathrm{V}_{20}$ by $1.1 \%, 0.97 \%$, and $0.95 \%$ for the 3DCRT plans, respectively, and overestimated $\mathrm{V}_{5}, \mathrm{~V}_{10}$, and $\mathrm{V}_{20}$ by $1.95 \%, 1.6 \%$, and $1.72 \%$ for the IMRT plans, respectively. The PBC overestimated $\mathrm{V}_{20}$ by $1.49 \%$ but underestimated $\mathrm{V}_{5}$ by $2.7 \%$ for 3DCRT, the difference in $\mathrm{V}_{10}$ was not statistically significant. The PBC overestimated the average $\mathrm{V}_{10}$ and $\mathrm{V}_{20}$ by $1.44 \%$ and $1.78 \%$, respectively for the IMRT plans but underestimated the average $\mathrm{V}_{5}$ by $1.34 \%$.

\section{Discussion}

In this study, we used 24 patients to investigate the effects of the dose calculation accuracy on plan evaluation for lung cancer treatments. Both based on the statistical analysis and the evaluation of all individual patient cases, it is clear that large discrepancies occur between the different dose calculation algorithms. Therefore, substantial deviations will occur when an insufficiently accurate dose computation algorithm is selected. For lung cancer treatment planning, it is very important to consider the differences in tissue density during dose calculation and to accurately model secondary electron transport [13,22]. If the lower attenuation of photon beams within lung tissue is not considered, the dose to the tissues downstream will be underestimated. Furthermore, as the electron path length within the lung extends to several centimetres, the beam penumbra widens, larger volumes of the lung are exposed to significant doses and the dose near the beam edge decreases [23,24]. Additionally, an unbalance between the number of produced and absorbed electrons arses near the interface between the low and high density tissues causes the dosage to build up [25-27]. If the electronic disequilibrium effect is not considered, it will again cause an underestimation of the dose to the lung. These effects are expected to become more pronounced for smaller field sizes, higher photon energies, and decreased lung densities $[25,28,29]$.

The CCC is a superposition method based on a point kernel convolution with a fixed number of different directions along which the energy is transported from each grid point in the patient [17]. It employs three-dimensional (3D) density scaling of their kernels for inhomogeneity [30], whereas PBC applies only a one-dimensional convolution along fan lines [31]. The inhomogeneity correction is performed by an Equivalent Path Length correction (EPL) (i.e., using effective depths) [17].

Regarding dose reporting, $\mathrm{PBC}$ algorithms calculated the dose-to-water $\left(D_{w}\right)$ while the MC and CCC results reported herein were dose-to-medium $\left(D_{m}\right)$. In lung tissue and soft tissue, the differences between $D_{w}$ and $D_{m}$ are only approximately $1 \%$ [8,32-34]. A direct comparison of the two dose calculation algorithms (PBC and

Table 2 Results of the statistical analysis for GTV and PTV, for the mean dose and every dose-volume point, the results of the paired $t$ test are reported

\begin{tabular}{|c|c|c|c|c|c|c|c|c|c|c|c|c|c|c|}
\hline & & & $\mathrm{D}_{\text {mean }}(\%)$ & $\mathbf{p}$ & $\mathrm{D}_{98}(\%)$ & $p$ & $\mathrm{D}_{95}(\%)$ & $p$ & $\mathrm{D}_{90}(\%)$ & $p$ & $\mathrm{D}_{50}(\%)$ & $p$ & $\mathrm{D}_{2}(\%)$ & $p$ \\
\hline \multirow[t]{4}{*}{ 3DCRT } & CCC vs. MC & GTV & 0.62 & 0.16 & 0.68 & 0.18 & 0.86 & 0.08 & 0.91 & 0.62 & 0.79 & 0.08 & 0.019 & 0.97 \\
\hline & & PTV & 0.42 & 0.32 & -0.39 & 0.40 & -0.25 & 0.61 & 0.05 & 0.91 & 0.53 & 0.22 & 0.013 & 0.98 \\
\hline & PBC vs. MC & GTV & 5.95 & 0.00 & 7.57 & 0.00 & 7.28 & 0.00 & 6.95 & 0.00 & 5.76 & 0.00 & 4.63 & 0.00 \\
\hline & & PTV & 7.96 & 0.00 & 12.51 & 0.00 & 11.18 & 0.00 & 10.04 & 0.00 & 7.61 & 0.00 & 5.85 & 0.00 \\
\hline \multirow[t]{4}{*}{ IMRT } & CCC vs. MC & GTV & 3.43 & 0.00 & 5.36 & 0.00 & 5.06 & 0.00 & 4.77 & 0.00 & 3.51 & 0.00 & 1.15 & 0.02 \\
\hline & & PTV & 3.05 & 0.00 & 3.37 & 0.00 & 3.39 & 0.00 & 3.50 & 0.00 & 3.27 & 0.03 & 2.16 & 0.03 \\
\hline & PBC vs. MC & GTV & 7.81 & 0.00 & 12.84 & 0.00 & 11.49 & 0.00 & 10.50 & 0.00 & 7.44 & 0.00 & 5.04 & 0.00 \\
\hline & & PTV & 10.23 & 0.00 & 20.76 & 0.00 & 18.07 & 0.00 & 15.9 & 0.00 & 9.4 & 0.00 & 5.41 & 0.00 \\
\hline
\end{tabular}

Note: $G T V$ = gross tumour volume; PTV = planning target volume; $M C=$ Monte Carlo; $C C C$ vs. $M C$ means $\left(D_{C C C}-D_{M C}\right) / D_{M C} P B C$ vs. $M C$ means $\left(D_{P B C}-D_{M C}\right) / D_{M C}$. 
Table 3 Results of the statistical analysis for ipsilateral lung, results of the paired $\mathbf{t}$ test are reported

\begin{tabular}{llllllll}
\hline & & $\mathbf{V}_{\mathbf{5} \text { Gy }}$ & $\mathbf{p}$ & $\mathbf{V}_{\mathbf{1 0} \text { Gy }}$ & $\mathbf{p}$ & $\mathbf{V}_{\mathbf{2 0} \text { Gy }}$ & $\mathbf{p}$ \\
\hline \multirow{2}{*}{ 3DCRT } & CCC vs. MC & 1.10 & 0.01 & 0.97 & 0.00 & 0.95 & 0.00 \\
& PBC vs. MC & -2.70 & 0.00 & 0.17 & 0.47 & 1.49 & 0.00 \\
IMRT & CCC vs. MC & 1.95 & 0.00 & 1.6 & 0.00 & 1.72 & 0.00 \\
& PBC vs. MC & -1.34 & 0.00 & 1.44 & 0.00 & 1.78 & 0.00 \\
\hline
\end{tabular}

Note: GTV = gross tumour volume; PTV = planning target volume; $\mathrm{MC}=$ Monte Carlo; $C C C$ vs. $M C$ means $V_{C C C}-V_{M C}$ PBC vs. $M C$ means $V_{P B C}-V_{M C}$.

$\mathrm{MC}$ ) is therefore possible without introducing a larger error into $D_{m}$ vs. $D_{w}$.

Differences have been found when comparing $\mathrm{PBC}$ and CCC with MC algorithms for lung cancer treatment. The results of this study show that the dose prediction of CCC is closer to $\mathrm{MC}$ than that of PBC. Our previous study [25] and other previous studies [6-9,18] all discovered that the quantification of the difference between $\mathrm{CCC}$ and $\mathrm{MC}, \mathrm{PBC}$ and $\mathrm{MC}$ depend on beam energy, lung density, target volume, target position and geometry.

In previous studies Dobler et al. presented the results of a comparison between $\mathrm{PB}, \mathrm{CC}, \mathrm{XVMC}$, and film measurement in one phantom case and indicated that there was a deviation of approximately $8 \%$ between CC and measurement/XVMC for beam energy of 6MV [7]. Huixiao Chen et al. compared the distributions of $\mathrm{PB}$, $\mathrm{XVMC}$, and film measurement for typical plan applied to inhomogeneous anthropomorphic phantom, and discovered that the deviation between $\mathrm{PB}$ and film measurement was up to $15 \%$. They also compared the dose calculation between PB and XVMC for 35 clinical cases, and revealed that the deviation of mean dose for PTV and GTV between PB and XVMC was approximately
$7 \%$ and $4 \%$, respectively [31]. Stephen F. Kry et al. retrospectively analyzed the results of 304 irradiations of the Radiological Physics Center (RPC) thorax phantom at 221 different institutions as part of credentialing for RTOG clinical trials. The results revealed that: PB algorithm overestimated the dose delivered to the centre of the target by $4.9 \%$ on average; convolution/superposition (CS) algorithms also showed a systematic overestimation of the dose to the centre of the target by $3.7 \%$ on average; in contrast, the $\mathrm{MC}$ algorithm dose calculations agreed with measurement within $0.6 \%$ on average [35]. For the most part, these studies are consistent with our results.

In general, the $\mathrm{MC}$ calculation is considered more reliable than the SC calculation. However, it should be noted that the $\mathrm{MC}$ calculation has an intrinsic deviation arising from statistical accuracy. This uncertainty is larger in lowdose regions.

A discrepancy between $\mathrm{PBC}$ and $\mathrm{MC}, \mathrm{CCC}$ and $\mathrm{MC}$ maybe involve the uncertainty arising from both the beam modeling and CT-to-density curves used in MC calculation. MC uses the physical density as the user input, whereas Oncentra Masterplan uses the electron density.

\section{Conclusions}

We compared the Monte Carlo algorithm with two commercial treatment planning algorithms (CCC and $\mathrm{PBC})$ for 24 lung cancer patients. The CCC algorithm overestimates the mean dose to the tumour by approximately $3 \%$ in the IMRT but is very close to the MC simulation in 3DCRT. The PBC overestimates the dose to the tumour in both 3DCRT and IMRT. Therefore, it is recommended that the treatment plan for lung cancer should be calculated using an advanced dose calculation algorithm other than the PBC algorithm. In IMRT, we should be pay more attention to
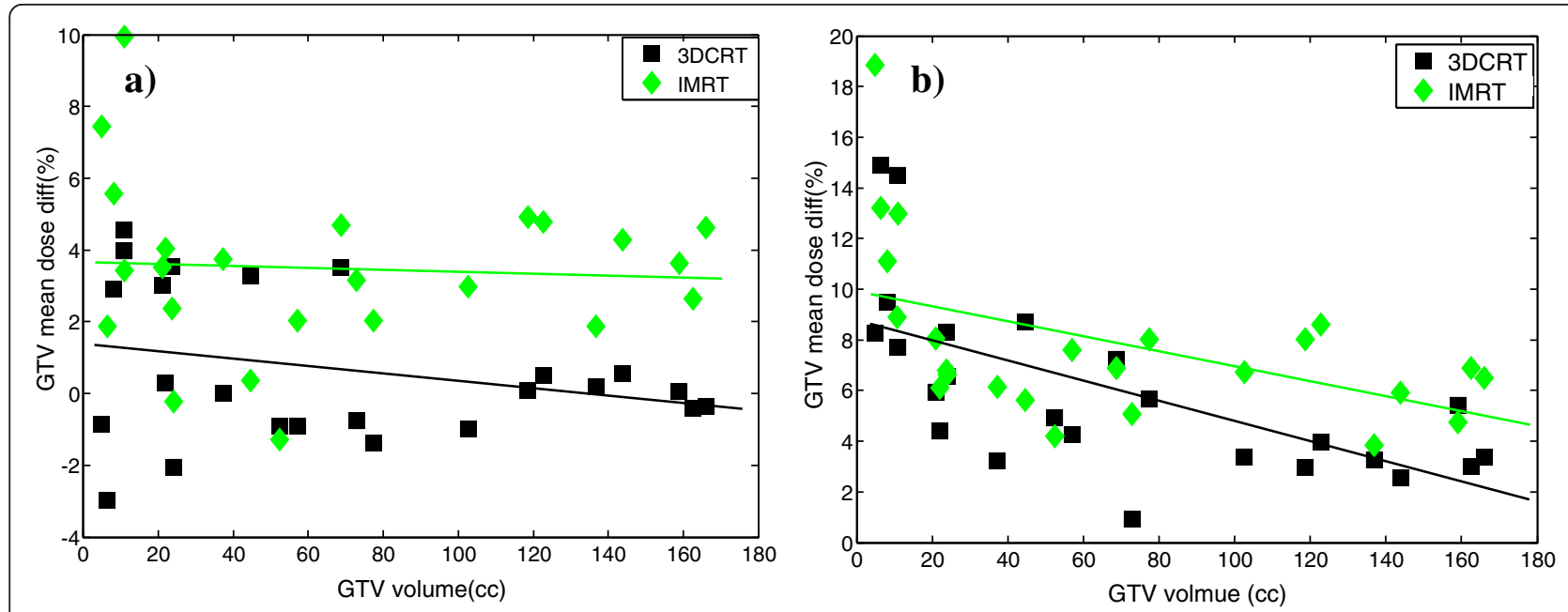

Figure 4 Mean dose differences of GTV between a) CCC and MC b) PBC and MC for all individual patients. 
the minimum segment width when setting up the optimisation parameters, if the minimum segment width is too small, it will probably bring lager uncertainty. To enhance the calculation accuracy, when commissioning a treatment planning system, one should attach great importance to the similarity between the calculations and the measurements for small fields, rather than focusing on large fields [6]. In our study, the Monte Carlo dose calculation results were quite consistent with the measurements. Therefore, if the Monte Carlo code had to be benchmarked before clinical use, the MC can provide a very good tool for benchmarking the performance of other dose calculation algorithms within patients (where measurements are difficult or even impossible) [13].

\section{Competing interests}

The authors declare that they have no competing interests.

\section{Authors contributions}

Yanqun Zhao, Pei Wang, Jian Li participated in the design of the study and drafted the manuscript. Gang yin, Xianliang Wang, Shengwei Kang carried out the Monte Carlo simulations. Mingyong Xiao, Jie Li, Guohai Qi, Xiongfei Liao performed the dose measurements, participated in the statistical analysis. All authors read and approved the final manuscript.

\section{Acknowledgments}

The authors would like to thank the Fox Chase Cancer Center, and specifically Mr. C-M Ma and Mr. J S Li, for supplying us with the MCSIM Monte Carlo code.

Received: 3 July 2014 Accepted: 4 December 2014

Published online: 16 December 2014

\section{References}

1. Papanikolaou N, Battista J, Boyer A, Kappas C, Klein E, Mackie T, Sharpe M, Van Dyk J: Tissue inhomogeneity corrections for megavoltage photon beams. AAPM Report No. 85, Task Group No 65 of the Radiation Therapy Committee of the American Association of Physicists in Medicine. Madison, WI: Medical Physics Publishing; 2004.

2. Vinall AJ, Williams AJ, Currie VE, Currie A, Van Esch A, Huyskes D: Practical guideline for routine intensity-modulated radiotherapy verification: pre-treatment verification with portal dosimetry and treatment verification with in vivo dosimetry. Br J Radiol 2010, 83(995):949 957.

3. International Commission of Radiation Units and Measurements: ICRU Report No.83: Prescribing, recording, and reporting intensity-modulated photon-beam therapy (IMRT). Bethseda: International Commission of Radiation Units and Measurements; 2010

4. Ramon Alfredo C. Siochi, Andrea Molineu: Patient-specific QA for IMRT should be performed using software rather than hardware methods Med Phys 2013, 40(7):070601-1- 0706013

5. Nelms BE, Zhen HM, Tome WA: Per-beam planar IMRT QA passing rates do not predict clinically relevant patient dose errors. Med Phys 2011, 38(2):1037 1043

6. Wilcox EE, Daskalov GM, Pavlonnis G, Shumway R, Kaplan B, VanRoov E: Dosimetric verification of intensity modulated radiation therapy of 172 patients treated for various disease sites: Comparision of EBT film dosimetry, ion chamber measurements and independent MU calculation. Med Dosim 2008, 33(4):303 309.

7. Dobler B, Walter C, Knopf A, Fabri D, Loeschel R, Polednik M, Schneider F, Wenz F, Lohr F: Optimization of extracranial stereotactic radiation therapy of small lung lesions using accurate dose calculation algorithms. Radiat Oncol 2006, 1:45.

8. Reynaert N, Van der Mark SC, Schaart DR, Xan Der Zee W, Van VlietVroegindeweij C, Tomsej M, Jansen J, Heijmen B, Coghe M, De Wagter C: Monte Carlo treatment planning for photon and electron beams. Radiat Phys Chem 2007, 76(4):643 686.
9. Carrasco P, Jornet N, Duch MA, Weber L, Ginjaume M, Eudaldo T, Jurado D, Ruiz A, Ribas M: Comparison of dose calculation algorithms in phantom with lung equivalent heterogeneities under conditions of lateral electronic disequilibrium. Med Phys 2004, 31(10):2899 2911.

10. Koelbl O, Krieger T, Haedinger U, Sauer O, Flentje M: Influence of calculation algorithm on dose distribution in irradiation of non-small cell lung cancer (NSCLC) collapsed cone versus pencil beam. Strahlenther Onkol 2004, 180(12):783 788.

11. Haedinger $U$, Krieger T, Flentje M, Wulf J: Influence of calculation model on dose distribution in stereotactic radiotherapy for pulmonary targets. Int J Radiat Oncol Biol Phys 2005, 61(1):239 249.

12. Krieger T, Sauer OA: Monte Carlo- versus pencil-beam-/collapsed-cone-dose calculation in a heterogeneous multi-layer phantom. Phys Med Biol 2005, 50(5):859 868 .

13. Vanderstraeten B, Reynaert N, Paelinck L, Madani I, De Wagter C, De Gersem W, De Neve W, Thierens H: Accuracy of patient dose calculation for lung IMRT: a comparison of Monte Carlo, convolution/superposition, and pencil beam computations. Med Phys 2006, 33(9):3149 3158.

14. Seco J, Adams E, Bidmead M, Partridge M, Verhaegen F: Head-and-neck IMRT treatments assessed with a Monte Carlo dose calculation engine. Phys Med Biol 2005, 50(5):817 830.

15. Wang L, Yorke E, Chui CS: Monte Carlo evaluation of 6 MV intensity modulated radiotherapy plans for head and neck and Lung treatments. Med Phys 2002, 29(11):2705 2717.

16. Yang J, Li J, Chen L, Price R, McNeeley S, Qin L, Wang L, Xiong W, Ma CM: Dosimetric verification of IMRT treatment planning using Monte Carlo simulation for prostate cancer. Phys Med Biol 2005, 50(5):869 878.

17. Aarup LR, Nahum AE, Zacharatou C, Juhler-Nttrup T, Kns T, Nystrm H, Specht L, Wieslander E, Korreman SS: The effect of different lung densities on the accuracy of various radiotherapy dose calculation methods: Implications for tumour coverage. Radiother Oncol 2009, 91(3):405 414.

18. Kawrakow I, Rogers D: The EGSnrc code system:Monte Carlo simulation of electron and photon transport, NRCC report PRIS.701. Ottawa: Ionizing radiation standards national research council of Canada; 2010.

19. Walters S, Kawrakow, Rogers DWO, Rogers: DOSXYZnrc users manual, NRCC report PRIS-794. Ottawa: Ionizing radiation standards national research council of Canada; 2009

20. Ma CM, Li JS, Pawlicki T, Jiang SB, Deng J, Lee MC, Koumrian T, Luxton M, Brain S: A Monte Carlo dose calculation tool for radiotherapy treatment planning. Phys Med Biol 2002, 47(10):1671 1689.

21. Aljarrah K, Pawlickj T, Niemierko A: A clinical study of MLC-based IMTT lung dose calculation accuracy on plan evaluation parameters. $J$ Cancer Sci Ther 2010, 2(3):074 081.

22. De Neve W, De Wagter C: Lethal pneumonitis in a phase I study of chemotherapy and IMRT for NSCLC: the need to investigate the accuracy of dose computation. Radiother Oncol 2005, 75(2):246 247.

23. Kornelsen $\mathrm{RO}$, Young ME: Changes in the dose-profile of a10 MV x-ray beam within and beyond low density material. Med Phys 1982, 9(1):114 116.

24. Miller RC, Bonner JA, Kline RW: Impact of beam energy and field margin on penumbra at lung tumor-lung parenchyma interfaces. Int J Radiat Oncol Biol Phys 1998, 41(3):707 713

25. Zhao Y-q, Wang P, Li J, Xiao M-y, Lang Jin-yi Wu D-k: Compare the calculation precision of collapsed cone convolution and pencil beam convolution algorithm in heterogeneous tissue. Chin J Radiat Oncol 2012, 21(1):72 76.

26. Werner BL, Das IJ, Khan FM, Meigooni AS: Dose perturbations at interfaces in photon beams. Med Phys 1987, 14(4):585 595.

27. Mijnheer BJ, Rice RK, Chin LM: Lead-polystyrene transition zone dosimetry in high-energy photon beams. Radiother Oncol 1988, 11(4):379 386.

28. Mackie TR, El-Kathib E, Battista J, Scrimger J, Van Dyk J, Cunningham JR: Lung dose corrections for 6- and 15-MV x rays. Med Phys 1985, 12(3):327 332.

29. Rice RK, Mijnheer BJ, Chin LM: Benchmark measurements for lung dose corrections for X-ray beams. Int J Radiat Oncol Biol Phys 1988, 15(2):399 409.

30. Ahnesj A: Collapsed cone convolution of radiant energy for photon dose calculation in heterogeneous media. Med Phys 1989, 16(4):577 592

31. Ahnesj A, Saxner M, Trepp A: A pencil beam model for photon dose calculation. Med Phys 1992, 19(2):263 273.

32. Chen H, Lohr F, Fritz P, Wenz F, Dobler B, Lorenz F, Mhlnickel W: Stereotactic, single-dose irradiation of lung tumors: a comparison of absolute dose and dose distribution between pencil beam and monte carlo algorithms based on actual patient CT scan. Int J Radiat Oncol Biol Phys 2010, 78(3):955 963. 
33. Liu HH, Keall P: Dm rather than Dw should be used in Monte Carlo treatment planning. Med Phys 2002, 29(5):922 923.

34. Siebers JV, Kell PJ, Nahum AE, Mohan R: Converting absorbed dose to medium to absorbed dose to water for Monte Carlo, based photon beam dose calculations. Phys Med Bio 2004, 45(4):983 995.

35. Kry SF, Alvarez P, Molineu A, Amador C, Galvin J, Followill DS: Algorithms used in heterogeneous dose calculations show systematic differences as measured with the radiological physics centers anthropomorphic thorax phantom used for RTOG credentialing. Int I Radiat Oncol Biol Phys 2013, 85(1):e95 e100.

doi:10.1186/s13014-014-0287-2

Cite this article as: Zhao et al:: A clinical study of lung cancer dose calculation accuracy with Monte Carlo simulation. Radiation Oncology 2014 9:287.

\section{Submit your next manuscript to BioMed Central and take full advantage of:}

$\otimes$ Convenient online submission

$\otimes$ Thorough peer review

$\nabla$ No space constraints or color $\nabla$ gure charges

$\nabla$ Immediate publication on acceptance

Q Inclusion in PubMed, CAS, Scopus and Google Scholar

$\otimes$ Research which is freely available for redistribution 